support of African farmers will help in the long run.

Fifth, investments in efforts to combat the global epidemics of AIDS and tuberculosis are much more generous than they were five years ago, but funding must be increased and sustained if we are to slow these increasingly complex epidemics. One of the most ominous recent developments is the advent of highly drug-resistant strains of both causative pathogens. "Extensively drug-resistant tuberculosis" has been reported in the United States, Eastern Europe, Asia, South Africa, and elsewhere; in each of these settings, the copresence of HIV has amplified local epidemics of these almost untreatable strains. Drug-resistant malaria is now common worldwide, extensively drug-resistant HIV disease will surely follow, and massive efforts to diagnose and treat these diseases ethically and effectively will be needed. We have already learned a great deal about how best to expand access to second-line antituberculous drugs while increasing control over their use ${ }^{5}$; these lessons must be applied in the struggles against AIDS, malaria, and other infectious pathogens.

Finally, there is a need for a renewed basic-science commitment to vaccine development, more reliable diagnostics (the 100-yearold tests widely used to diagnose tuberculosis are neither specific nor sensitive), and new classes of therapeutics. The research-based pharmaceutical industry has a critical role to play in drug development, even if the overall goal is a segmented market, with higher prices in developed countries and generic production with affordable prices in developing countries.

There has been a heartening increase in basic-science investments for tuberculosis and malaria; funding for HIV research at the National Institutes of Health remains robust. Yet the fruits of such research will not arrive in time for those now living with, and dying from, AIDS and tuberculosis. New tools to prevent, diagnose, and treat the diseases of poverty will be added to the stockpile of other potentially lifesaving products that do not reach the poorest people, unless we develop an equity plan to provide them. Right now, our focus must be on improving access to the therapies that are available in high-income countries. The past few years have shown us that we can make these services available to millions, even in the poorest reaches of the world.

The unglamorous and difficult process of increasing access to prevention and care needs to be our primary focus if we are to move toward the lofty goal of equitably distributed medical services in a world riven by inequality. Without such goals, the slogan "One World, One Hope" will remain nothing more than a dream.

An interview with Dr. Farmer can be heard at www.nejm.org.

Dr. Kim is chief of the Division of Social Medicine and Health Inequalities at Brigham and Women's Hospital, and Dr. Farmer is a professor of social medicine at Harvard Medical School; both are former directors of Partners in Health, all in Boston.

1. Walensky RP, Paltiel AD, Losina E, et al. The survival benefits of AIDS treatment in the United States. J Infect Dis 2006;194:11-9.

2. Walton DA, Farmer PE, Lambert W, Léandre F, Koenig SP, Mukherjee JS. Integrated HIV prevention and care strengthens primary health care: lessons from rural Haiti. J Public Health Policy 2004;25:137-58.

3. Behforouz HL, Farmer PE, Mukherjee JS. From directly observed therapy to accompagnateurs: enhancing AIDS treatment outcomes in Haiti and in Boston. Clin Infect Dis 2004; 38:Suppl 5:S429-S436.

4. Paton NI, Sangeetha S, Earnest A, Bellamy R. The impact of malnutrition on survival and the CD4 count response in HIV-infected patients starting antiretroviral therapy. HIV Med 2006;7:323-30.

5. Gupta R, Kim JY, Espinal MA, et al. Responding to market failures in tuberculosis control. Science 2001;293:1049-51.

\title{
Changing the Paradigm for HIV Testing - The End of Exceptionalism
}

\author{
Ronald Bayer, Ph.D., and Amy L. Fairchild, Ph.D., M.P.H.
}

The Centers for Disease Control and Prevention (CDC) is poised to issue new recommendations for testing for HIV in adults, adolescents, and pregnant women. Frustrated that more than 25 percent of Americans with HIV infection are unaware of their status and that almost 40 percent of those with newly diagnosed AIDS discover that they are infected less than a year before diagnosis, officials have proposed that HIV screening be routinely offered in all health care settings.

The CDC already recommends routine testing among high-risk groups and in high-prevalence set- tings. The radical departure is the extension of routine testing to the entire population and the reconceptualization of the requirements for consent. Patients would be told that HIV testing was a routine part of care and given the opportunity to opt out. According to the CDC, specific signed consent would no 
longer be required, because "general consent for medical care is sufficient to encompass consent for HIV testing."

These proposals are similar to those offered by New York City's commissioner of health, Thomas Frieden, who has called for eliminating the requirements for pretest counseling and written informed consent that have been in place since 1988 and replacing the requirement for consent with the right to opt out. In a recent circular about informed consent, the New York City health department recommended that physicians say to their patients, in advising them about all the blood tests that will be performed, "I am going to . . . do an HIV test. Do you have any questions?"1 Inevitably, the effects of such challenges to the status quo will be felt throughout the country, in health departments, hospitals, and other clinical settings.

These moves signal the end of the exceptionalism that has distinguished public health policy with regard to AIDS from approaches to other communicable and sexually transmitted diseases. ${ }^{2}$ Against a backdrop of fear, stigma, and relative clinical powerlessness, AIDS activists and their public health allies initially sought approaches that would respect the autonomy and privacy rights of people with or at risk for HIV infection and that would offer protection from unwarranted discrimination. Avoiding compulsory measures such as isolation and quarantine, which were so much a part of the public health tradition, was all the more crucial, since the people with increased risk - gay and bisexual men, drug users, and their sexual partners - were already socially vulnerable. As then-Surgeon General C. Everett Koop often said, poli- cies and practices that appeared to threaten such persons could only drive the epidemic underground and make it more difficult to work with the populations within which HIV was spreading.

The exceptionalist perspective was reflected in policies on surveillance and partner notification, but it was strongest in the context of HIV testing. From 1985, when the HIV antibody test became available, fear about discrimination and stigmatization, concern about the potentially severe psychological burden of an HIV diagnosis in the absence of effective therapy, and skepticism about the relationship between testing and the changing of risky behavior led AIDS activists to warn about the dangers of the test. Public health officials, in contrast, saw the voluntary testing and counseling of people at high risk as the core feature of a preventive public health strategy. Out of the ensuing bitter conflict emerged some exacting standards for specific written consent and requirements for pretest counseling. This approach was markedly different from what typically happened in clinical settings, where physicians ordered blood work for patients who were generally unaware of what was being tested for and consent was assumed.

In the late 1980 s, as clinicians gained greater confidence in their ability to manage HIV disease, they began to bridle under the exacting restrictions that had been imposed a few years earlier. But it was in the cases of infants and pregnant women that exceptionalism was most directly challenged. Some pediatricians asserted that babies had a "right to be tested" because, if infected, they required vigilant medical care before they became sick - a right that trumped the mother's right to privacy, which would be breached by the discovery of maternal antibody in the newborn. Ultimately, two states - New York and Connecticut - enacted statutes mandating HIV testing in newborns.

More sustained were the efforts that followed the 1994 discovery that administering zidovudine during pregnancy could reduce the rate of vertical transmission by two thirds. In 1996, the House of Delegates of the American Medical Association passed a resolution recommending mandatory testing of pregnant women. Although such moves stalled, efforts to loosen the consent requirements for pregnant women gained traction. In 1998, the Institute of Medicine (IOM) recommended the routine testing of all pregnant women in the United States, calling for a shift from stringent consent to an informed right of refusal. ${ }^{3}$ A year later, the American Academy of Pediatrics and the American College of Obstetricians and Gynecologists jointly endorsed universal opt-out screening for pregnant women. When the CDC considered these recommendations in 2001, it endorsed universal screening of pregnant women. ${ }^{4}$ But while calling for a simplified "pretest process," eliminating timeconsuming counseling, it did not explicitly recommend an opt-out approach. ${ }^{4,5}$

By 2004, according to the Kaiser Family Foundation, four states Texas, Michigan, Tennessee, and Arkansas - required the testing of pregnant women unless they refused. In 13 states - including California, Florida, New Jersey, and Maryland - providers were required to offer pregnant women testing. Thus, the changes being proposed by the CDC and in New York grow out of more than a decade of efforts to loosen the strictures of HIV exceptionalism. 
The move to make testing more routinely available, or even to require that physicians offer it to patients, has encountered little opposition. But there is resistance to the elimination of pretest counseling and specific written consent, as well as to the adoption of an opt-out standard. Although Frieden rejects mandatory HIV testing, many fear that without a requirement for written consent, testing would in effect become compulsory. "This is not informed consent, and it is not even consent," said one activist. It is rather an attempt "to ram HIV testing down people's throats without their permission."

According to advocates of change, the transformation of HIV disease into a complex chronic condition requiring long-term, ongoing clinical management means that the limits imposed when medicine had little to offer have outlived their justification. Proponents argue that prevailing requirements impede wide-scale testing because they are burdensome and time-consuming. Furthermore, they relieve physicians of an obligation to offer testing. Less often acknowledged is the fact that an opt-out approach shifts the burden from those who would choose to undergo the test to those who would refuse.

Making it more difficult to say no may be justified by public health considerations, particularly by concern about preventable opportunistic infections in persons who are unaware of their HIV infection and about transmission to their sexual or needle-sharing partners. As state and local health policymakers consider the new CDC recommendations, it will be crucial that the assumptions both spoken and unspoken - informing the new testing paradigm be the subject of open, ethically informed, and evidence-based discussion.

Dr. Bayer is a professor and Dr. Fairchild an associate professor at the Center for the History and Ethics of Public Health, Department of Sociomedical Sciences, Mailman School of Public Health, Columbia University, New York.

1. Henn M, Malave M, Renaud T, Sepkowitz $\mathrm{K}$, Kellerman S. Making HIV testing a routine part of medical care. City Health Information. Vol. 25. No. 2. February 2006:9-12. (New York: New York City Department of Health and Mental Hygiene.) (Also available at http://www.nyc.gov/html/doh/downloads/ pdf/chi/chi25-2.pdf.)

2. Bayer R. Public health policy and the AIDS epidemic: an end to HIV exceptionalism? N EnglJ Med 1991;324:1500-4.

3. Stoto MA, Almario DA, McCormick MC, eds. Reducing the odds: preventing perinatal transmission of HIV in the United States. Washington, D.C.: National Academy Press, 1999.

4. Revised guidelines for HIV counseling, testing, and referral. MMWR Recomm Rep 2001;50 (RR-19):1-57.

5. Revised recommendations for HIV screening of pregnant women. MMWR Recomm Rep 2001;50(RR-19):63-85.

\section{Getting Serious about Cholera}

David A. Sack, M.D., R. Bradley Sack, M.D., Sc.D., and Claire-Lise Chaignat, M.D., M.P.H.

$\mathrm{D}$ eaths from cholera are again making news, this time in Angola. According to the World Health Organization (WHO), Angola had reported 46,758 cases of cholera, including 1893 deaths, as of June $19,2006 .{ }^{1}$ The outbreak has affected 14 of 18 provinces, but nearly half the cases were reported in the coastal capital, Luanda, and another 17 percent in Benguela provinces. The overall case fatality rate is about 4 percent, although in some provinces, it has reached 30 percent. This outbreak represents another in a series of cholera epidemics in this country, ${ }^{2}$ which is among the poorest in the world and is still recovering from years of armed conflict. Several other African countries have also had recent outbreaks (see map), in the seventh pandemic of cholera caused by Vibrio cholerae $\mathrm{O} 1 \mathrm{El}$ Tor, which reached Africa in 1970.

In addition to the reported cases, many are either not reported or are labeled as "acute watery diarrhea." Although 94 percent of the 101,383 cholera cases and 99 percent of the 2345 related deaths registered with the WHO in 2004 occurred in sub-Saharan Africa, these numbers present a misleading picture of the global cholera burden. $V$. cholerae infects persons in many developing countries, with the highest rates in Asia, but many Asian countries do not report their cases because of the effects on trade and travel, however unwarranted. The true burden is probably several million cases in Asia and Africa, with fewer cases in Latin America. Assuming a case fatality rate of 4 percent (on the basis of treated cases), annual mortality of at least 40,000 to 100,000 is probable, and even this may be an underestimation: since many patients die before reaching a health care facility, the true number of deaths may be much higher.

In the 21st century, we know a great deal about cholera - its ecology, transmission patterns through contaminated water, and pathophysiology. ${ }^{3}$ Simple, inexpensive rehydration treatment is nearly 100 percent successful, and safe oral vaccines can prevent most 